\title{
Translation and validation of the Child Three-Factor Eating Questionnaire (CTFEQr17) in French-speaking Canadian children and adolescents
}

\author{
Isabelle Frappier ${ }^{1,2,3,4}$ (0), Raphaëlle Jacob 1,2,3,4, Shirin Panahi 1,3,4,5,6, \\ David Larose $1,3,4,6$, Eleanor J Bryant ${ }^{7}$, Jean-Philippe Chaput ${ }^{8}$, David Thivel ${ }^{9}$ and \\ Vicky Drapeau $1,3,4,5, *$ \\ ${ }^{1}$ Centre NUTRISS, Institute of Nutrition and Functional Foods, Université Laval, Québec City, QC, Canada: ${ }^{2}$ School of \\ Nutrition, Université Laval, Québec City, QC, Canada: ${ }^{3}$ Québec Heart and Lung Institute Research Centre, Université \\ Laval, Québec City, QC, Canada: ${ }^{4}$ Centre de Recherche Interuniversitaire sur la Formation et la Profession \\ Enseignante (CRIFPE), Université Laval, Québec City, QC, Canada: ${ }^{5}$ Department of Physical Education, Université \\ Laval, 2300, Rue de la Terrasse, Québec City, QC, Canada GIV OA6: 'Department of Kinesiology, Université Laval, \\ Québec City, QC, Canada: ${ }^{7}$ Faculty of Social Sciences, University of Bradford, Bradford, UK: ${ }^{8}$ Healthy Active Living \\ and Obesity Research Group, Children's Hospital of Eastern Ontario Research Institute, Ottawa, Canada: ${ }^{9}$ Laboratory \\ of the Metabolic Adaptations to Exercise under Physiological and Pathological Conditions, Clermont Auvergne \\ University, Clermont-Ferrand, France
}

Submitted 14 February 2021: Final revision received 29 August 2021: Accepted 3 September 2021: First published online 10 September 2021

\begin{abstract}
Objective: To translate and validate the Child Three-Factor Eating Questionnaire (CTFEQr17), assessing cognitive restraint (CR), uncontrolled eating (UE) and emotional eating (EE), among French-speaking Canadian young individuals.

Design: Phase 1 comprised a translation and the evaluation of the comprehension of the questionnaire. Phase 2 comprised a confirmatory factor analysis (CFA), the evaluation of internal consistency (Cronbach's $\alpha$ ), test-retest reliability (intra-class correlation coefficients (ICC)) and construct validity, including correlations among the CTFEQr17 and Eating Attitudes Test (EAT-26), anthropometrics, dietary intake and diet quality.

Setting: Primary and secondary schools, Québec City, Canada.

Participants: Phases 1 and 2 included 20 (40\% boys, mean age 11.5 (SD 2.4) years) and 145 ( $48 \%$ boys, mean age 11.0 (SD 1.9) years) participants, respectively.

Results: Phase 1 resulted in the questionnaire to be used in Phase 2. In Phase 2, the CFA revealed that the seventeen item, three-factor model (CTFEQr17) provided an excellent fit. Internal consistency was good (Cronbach's $\alpha$ : 0.81-0.90). Test-retest reliability was moderate to good (ICC $=0.59$, (95\% CI 0.48, 0.70), ICC $=0 \cdot 78,(95 \%$ CI $0 \cdot 70,0 \cdot 84)$, ICC $=0.50,(95 \%$ CI $0 \cdot 38,0.62)$ for CR, UE and EE, respectively). CR correlated with EAT-26 score $(r=0.43, P<0.0001)$. UE and EE correlated negatively with BMI $Z$-scores ( $r=-0 ; \cdot 26, P=0 \cdot 003 ; r=-0 ; \cdot 19, P=0 \cdot 03$, respectively). CR correlated with the proportion of energy intake from protein and diet quality ( $r=0 \cdot 18, P=0.04 ; r=0 \cdot 20, P=0.02$, respectively).

Conclusion: The CTFEQr17 is suitable to use among French-speaking Canadian young individuals.
\end{abstract}

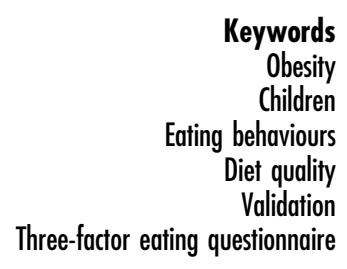

In Canada, overweight and obesity affect nearly one in three children ${ }^{(1)}$. Since children with obesity are at greater risk of obesity and related complications in adulthood ${ }^{(2-5)}$, it is important to focus on strategies to prevent long-term consequences associated with body weight gain ${ }^{(3,4)}$.
While the causes of childhood obesity are complex ${ }^{(6,7)}$, eating behaviour traits have been suggested to influence body weight and obesity among adults ${ }^{(8)}$. Moreover, eating behaviours have been shown to partially mediate genetic susceptibility to obesity in adults ${ }^{(9)}$. Among eating 
behaviours, disinhibition (i.e. overconsumption of food in response to cognitive or emotional cues) and susceptibility to hunger (i.e. food intake in response to feelings and perceptions of hunger) have been correlated with a greater risk of overweight and obesity in adults in the Québec Family Study ${ }^{(10)}$. High cognitive restraint (CR) level (i.e. conscious efforts to limit food intake to control or lose body weight) was also correlated with greater weight gain after 6 years of follow-up ${ }^{(11)}$. In children, eating behaviours have been shown to predict changes in weight status over time ${ }^{(12)}$. In adolescents with obesity, CR has been correlated with greater ad libitum energy intake (EI) following a weight loss program, potentially favouring weight regain ${ }^{(13)}$.

In adults, eating behaviours are widely assessed with the Three-Factor Eating Questionnaire (TFEQ) ${ }^{(14)}$ that measures three dimensions of eating behaviours: CR, disinhibition and susceptibility to hunger ${ }^{(14)}$. Two shortened versions of this questionnaire were subsequently developed, resulting in the TFEQ-R18 ${ }^{(15)}$ and TFEQ-R21 ${ }^{(16)}$. Both versions include the measurement of $\mathrm{CR}$ which was retained from the original TFEQ, uncontrolled eating (UE) (i.e. tendency to overeat and eat in response to multiple stimuli) which results in the combination of disinhibition and hunger factors from the original TFEQ, and emotional eating (EE) (i.e. eating in response to negative emotions) which emerges from some disinhibition items $^{(15,16)}$. The TFEQ-R21 questionnaire was later validated in an adult population and refined, resulting in the TFEQR18V2 ${ }^{(17)}$. Although this latter study found only small correlations with eating behaviour traits and $\mathrm{BMI}^{(17)}$, other studies in adult populations have shown that $\mathrm{UE}^{(18,19)}, \mathrm{EE}$ and $\mathrm{CR}^{(19,20)}$ were correlated with a higher BMI. The same correlations have been found in a sample of young females ${ }^{(21)}$. In children populations, eating behaviour traits have also been correlated with BMI and BMI $Z$-score ${ }^{(22-27)}$ and food preferences $^{(25,26)}$. However, little is known about the relationship between eating behaviours, obesity and diet in children and adolescents.

To better understand the importance of eating behaviour traits in relation to childhood obesity, researchers validated an English ${ }^{(25)}$ and Spanish ${ }^{(23)}$ version of the TFEQR21 adapted for children and adolescents. The validation study conducted by Bryant and colleagues resulted in a seventeen-item version of the questionnaire (CTFEQr17) and reported good internal consistency ${ }^{(25)}$. This questionnaire was also later validated in an English-Canadian sample, revealing a four-factor and twenty-item structure ${ }^{(26)}$, a Romanian sample, preserving the twenty-one-item original structure $^{(24)}$ and a Turkish sample, resulting in the same seventeen-item version as validated by Bryant and colleagues ${ }^{(27)}$. While it could represent an essential aspect of improving the treatment and prevention of childhood obesity, there is currently no validated French version of the CTFEQ.

This study aimed to translate and validate a French version of the Child Three-Factor Eating Questionnaire in a sample of French-speaking Canadian children and adolescents and also aimed to examine internal consistency reliability, test-retest reliability and construct validity with the correlations among eating behaviours and eating attitudes, anthropometric measures, dietary intake and diet quality. Regarding construct validity, we hypothesise that CR will be positively correlated with Eating Attitudes Test (EAT-26) score and negatively correlated with EI, waist circumference (WC) and BMI $Z$-score, and that UE and EE will be positively correlated with EI, WC and BMI $Z$-score.

\section{Methods}

\section{Phase 1: Translation of the CTFEQ and evaluation of the comprebension and acceptability of the questionnaire}

A French translation of the twenty-one-item CTFEQ developed by Bryant and colleagues ${ }^{(25)}$ was conducted and then back-translated into English by one individual with proficiency in both English and French and by a certified translator. Both English versions were compared, and the French version was adjusted to obtain a final French version. To ensure its understandability for children and adolescents, the French translation was evaluated by a child nutrition expert and by one paediatrician/researcher. Informal verification of the understanding of the questionnaire was conducted in a sample of three young individuals aged between 8 and 15 years, who provided verbal comments about their understanding of the questionnaire. Formal understanding and acceptability of the questionnaire were then assessed within a small group of young individuals recruited via an informal network of the research team ( $n 20$ participants aged $8-15$ years; mean age 11.5 (SD 2.4 ) years), through semi-structured interviews that were about $20 \mathrm{~min}$ in duration. Participants were asked to complete the questionnaire, to comment on each item and scale and to rephrase the instructions to identify misunderstood words or items. The interviewer completed an evaluation sheet identifying the elements, concepts or words that were not understood by the participants. The mean duration time of the questionnaire completion was 4.7 (SD 1.1) minutes. All items and scales were judged to be well understood by the participants; therefore, no item was modified or removed. This phase resulted in a final French version of the CTFEQ that was used in Phase 2.

\section{Phase 2: Validation of the CTFEQ}

\section{Participants}

Participants included 162 children and adolescents recruited from four primary and secondary schools in Québec City, Canada. School recruitment was conducted by contacting school principals by email. Participant recruitment was conducted via personal contact. Inclusion criteria were being aged between 8 and 15 years, francophone and having no significant learning difficulties 


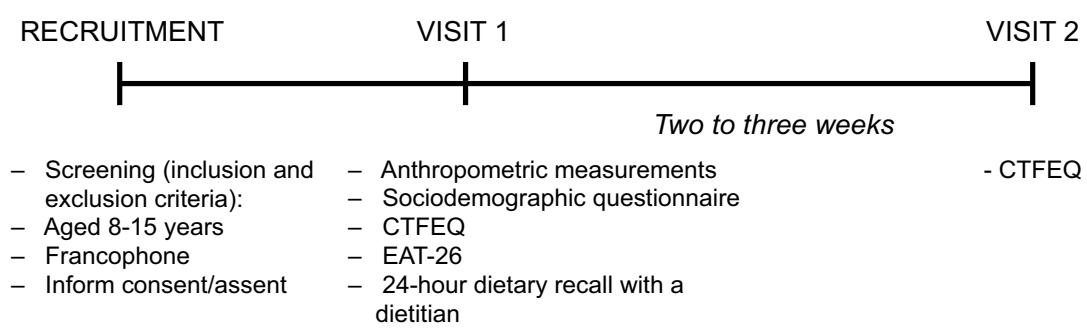

Fig. 1 Study protocol

(e.g. dyslexia), which was screened by teachers at schools before recruitment for participants. A total of 17 participants did not complete the study because they were absent from school during the data collection period. Thus, the final sample comprised of 145 individuals.

\section{Study protocol}

This study included two visits taking place in schools. During the first visit, anthropometric measurements were performed. Then, each participant completed questionnaires including the CTFEQ, and a 24-h dietary recall administered by a registered dietitian. Two to three weeks after the first visit, a second visit took place in schools to complete the CTFEQ a second time (Fig. 1).

\section{Measures}

\section{Sociodemographic characteristics}

A sociodemographic questionnaire was completed during the first visit to obtain information on the date of birth, sex, school grade and ethnicity of each participant.

\section{Child Three-Factor Eating Questionnaire (CTFEQ)}

The CTFEQ is a twenty-one-item self-reported questionnaire developed by Bryant and colleagues ${ }^{(25)}$, based on the ThreeFactor Eating Questionnaire-R21 (TFEQ-R21) ${ }^{(14)}$. This questionnaire assesses $\mathrm{CR}$, a six-item factor, UE, a nine-item factor and EE, a six-item factor. Items 1-20 are measured on a fourpoint Likert scale and item 21 is measured on an eight-point Likert scale, which is then converted into a four-point scale. Mean scores for each factor are calculated with the same coding procedure as presented by Bryant and colleagues ${ }^{(25)}$ : items 1-16 were reverse coded, items 17-20 were normally coded and item 21 was modulated into a four-point scale $(1-2=1 ; 3-4=2 ; 5-6=3 ; 7-8=4)$ (see online Appendix in Supplemental Material).

\section{Eating Attitudes Test (EAT-26)}

The French validated version of the EAT-26(28) , which is a self-administrated questionnaire, was administered. This questionnaire measures symptoms associated with eating disorders, including dieting (i.e. avoidance of fattening foods and preoccupation with being thinner), bulimia and food preoccupation (i.e. thoughts about food and thoughts indicating bulimia) and oral control (i.e. self-control of eating and perceived pressure from others to gain weight $)^{(29)}$. Participants obtaining a total score of over 20 are suggested to be at risk of having an eating disorder ${ }^{(29)}$.

\section{Anthropometrics}

Body weight was measured with light clothing and without shoes using a portable bioimpedance weighing scale (TANITA, model TBF-310) and recorded to the nearest $0 \cdot 1$ $\mathrm{kg}$. Height was obtained using a portable stadiometer and recorded to the nearest $0 \cdot 1 \mathrm{~cm}$. WC was measured midway between the lowest rib and the top of the iliac crest with a measuring tape to the nearest $0 \cdot 1 \mathrm{~cm}$. Two WC measures were taken, and the average was used in analyses. BMI was calculated as the weight $(\mathrm{kg})$ divided by height squared $\left(\mathrm{m}^{2}\right)$ and then converted into a BMI $Z$-score ${ }^{(30)}$. BMI $Z$-score was calculated according to the WHO procedure ${ }^{(31)}$, using the WHO anthropometric $Z$-scores 0-19 years calculator ${ }^{(32)}$. Children were then classified into four weight categories as follows: underweight (BMI $Z$-score $<-2$ ), normal weight $(1>$ BMI $Z$-score $\geq-2$ ), overweight ( $2>$ BMI $Z$-score $\geq 1$ ) or obese (BMI $Z$-score $\geq 2$ ).

\section{Dietary intake and diet quality}

One 24-h dietary recall was completed for each participant during the first visit by three registered dietitians. Detailed information on all foods and beverages consumed during the previous day was collected using the USDA five-step multiple-pass method ${ }^{(33,34)}$. To increase the accuracy of food portion estimations, food models and standard kitchen measures were used. Dietary recalls were analysed with Nutrific nutrient analysis software version 2015 (Université Laval, Québec, QC, Canada) which is based on the Canadian Nutrient File version $2015^{(35)}$, to obtain energy, macronutrient and micronutrient intakes. Two 24-h dietary recalls were excluded from the analysis due to the lack of information provided by the participants during the interviews. Diet quality was assessed using the Nutrient-Rich Food Index 9.3 (NRF 9.3), a nutritional quality index based on nine nutrients to encourage (i.e. protein, fibre, vitamins A, E and C, calcium, iron, potassium and magnesium) and three nutrients to limit (i.e. SFA, total sugars and sodium) ${ }^{(36,37)}$. A higher NutrientRich Food Index 9.3 score indicates a higher diet quality.

\section{Statistical analysis}

The complete database was examined for missing data. For each questionnaire, imputation was performed when there 
was a maximum of two missing items per factor per participant. Imputation procedure consisted in replacing the missing data with the mean value of its associated item. Mean data imputation was performed since it is an acceptable imputation method when proportions of missing data reach up to $10 \%{ }^{(38)}$ and that only small proportions of missing data were found in this study. For the CTFEQ questionnaire completed at the first and second visits, $1.3 \%$ and $0.6 \%$ of the data were missing, resulting in $25.0 \%$ and $92.9 \%$ of the missing data being imputed, respectively. For the EAT-26 questionnaire, $1.5 \%$ of data were missing and $39.3 \%$ were imputed. Prior to the analyses, distributions of the quantitative variables were evaluated based on the skewness and kurtosis indices and by visual inspection. Data were also examined for outliers with the JMP Outlier Analysis report. One outlier for the EAT-26 questionnaire was removed and two outliers for nutritional data were also removed for all statistical analysis.

\section{Descriptive analysis}

A descriptive analysis for participant characteristics was performed. Sex differences in participant characteristics were assessed using Student $t$-tests for continuous variables and $\chi^{2}$ tests for nominal variables.

\section{Factorial structure}

Confirmatory factor analysis using the maximum likelihood method with robust options for ordinal categorical variables was performed. The fit to the data was tested on three different models of the CTFEQ found in the literature to determine the most suitable factorial structure. Model 1 included the twenty-one-item model loading into three factors validated by Martín-García et $a{ }^{\left({ }^{(23)}\right.}$ and later validated by Steff et $a l{ }^{(24)}$. Model 2 included the seventeen-item model loading into three factors validated by Bryant et al. ${ }^{(25)}$. Model 3 included the twenty-item model loading into four factors validated by Yabsley et $a{ }^{(26)}$. The adequacy of the model fit was determined according to the different fit indices resulting from each factor analysis, using the thresholds proposed by $\mathrm{Hu}$ and Bentler ${ }^{(39)}$. A Non-Normed Fit Index $>0.95$; a Comparative Fit Index $>0.95$ and a Root Mean Squared Error of Approximation $\leq 0.06$ were considered adequate ${ }^{(39)}$. $\chi^{2}$ and normed chi-square values $\left(\chi^{2} / \mathrm{df}\right)$ were assessed, since regular $\chi^{2}$ values tend to be affected by sample size ${ }^{(40)}$. Values less than 5 and 2 indicate an acceptable and very good fit, respectively ${ }^{(41,42)}$. Standardised factor loadings were assessed in each confirmatory factor analysis. Akaike's Information Criteria (AIC) was also assessed, with smaller values of AIC indicating the most parsimonious model ${ }^{(43)}$.

\section{Reliability}

Cronbach's $\alpha$ coefficients based on polychoric correlations were used to evaluate internal consistency reliability for each factor of the models tested (i.e. adequate internal consistency as Cronbach's $\alpha \geq 0 \cdot 70)^{(42,43)}$. Intra-class correlation coefficients (ICC) were assessed with the Macro \% ICC9 for SAS two-way mixed-effects ${ }^{(44)}$ for each factor using data from the two completions of the questionnaire to determine test-retest reliability. The $95 \% \mathrm{CI}$ for each ICC was used to determine the level of reliability ${ }^{(45)}$. Values less than 0.50 show poor reliability, values between 0.50 and 0.75 show moderate reliability, values between 0.75 and 0.90 show good reliability and values above 0.90 show excellent reliability ${ }^{(45)}$.

\section{Construct validity}

Pearson's correlations were performed between the CTFEQr17 scores and age for boys and girls independently since sex was a significant covariate for CR and EE. Pearson's partial correlations were performed between the CTFEQr17 scores for each factor and eating attitudes, anthropometric measures, dietary intake and diet quality. All correlations were adjusted for sex and age. Interaction for sex, age and body weight status were assessed. Correlations assessing dietary intakes and diet quality were performed without and with adjustments for misreporting. Subjects that were under-reporters and over-reporters were identified using the calculating method suggested by Huang et al., taking into account reported EI and predicted total energy expenditure $(\text { TEE })^{(46)}$. Predicted TEE was assessed with the Institute of Medicine's equations for dietary reference intake ${ }^{(47)}$. Since this study did not evaluate the objective measurement of physical activity level, subjects were assigned with low active physical activity coefficient of 1.4 , as proposed by Huang et $a l .{ }^{(46)}$, which is supported by the fact that only $39 \%$ of Canadian children and youth meet the required physical activity recommendations ${ }^{(48)}$. Participants were classified as under, over and plausible reporters when the \% EI/TEE was lower, higher or within the 1 SD cutoff, respectively, for the following equation: $\left.\left.\pm 1 S D=\sqrt{\left(\mathrm{CV}_{\mathrm{rEI}}^{2}\right.} / d\right)+\mathrm{CV}_{\mathrm{pER}}^{2}+\mathrm{CV}_{\mathrm{mTEE}}^{2}\right), \quad$ in which $\mathrm{CV}_{\mathrm{rEI}}$ is the intra-variation of $\mathrm{EI}(\%)$, where a value of $23 \%{ }^{(49)}$ was used; $d$ is the number of recording days ( 1 in the present study); $\mathrm{CV}_{\mathrm{pER}}$ is the error of the parameters in the equations of predicted energy requirements, including physical activity level, where a value of $15 \%$ was obtained; and $\mathrm{CV}_{\text {mTEE }}$ is the day-to-day variation and measurement error for TEE based on doubly labelled water technique, where a value of $8 \cdot 2 \%$ was used $^{(46)}$. The cutoffs were exponentiated since EI distribution is skewed. Confirmatory factor analysis and Cronbach's $\alpha$ coefficients calculation were performed on EQS Multivariate Software, version 6.2. All the remaining analyses were performed on JMP software, version 14.0 from Statistical Analysis System (SAS Institute) and SAS software, version 9.4 (SAS Institute). Results are presented as mean (SD) or SD. Statistical significance was set at $P<0.05$.

\section{Sample size}

A minimum of 100 participants was necessary to guarantee the stability of the variance-covariance matrix in confirmatory analysis ${ }^{(50)}$. The number of participants needed for 
Table 1 Participant characteristics

\begin{tabular}{|c|c|c|c|c|c|c|c|}
\hline \multirow[b]{2}{*}{ Variable } & \multicolumn{2}{|c|}{ Total $(n=145)$} & \multicolumn{2}{|c|}{ Boys $(n=69)$} & \multicolumn{2}{|c|}{ Girls $(n=76)$} & \multirow[b]{2}{*}{$P^{\star}$} \\
\hline & $n$ & $\%$ & $n$ & $\%$ & $n$ & $\%$ & \\
\hline \multicolumn{8}{|l|}{ Age (years) $\dagger$} \\
\hline Mean & \multirow{2}{*}{\multicolumn{2}{|c|}{$\begin{array}{r}11.0 \\
1.9\end{array}$}} & \multicolumn{2}{|c|}{$10 \cdot 8$} & \multicolumn{2}{|c|}{$11 \cdot 3$} & \multirow{2}{*}{0.13} \\
\hline SD & & & & & & & \\
\hline \multicolumn{8}{|l|}{ Anthropometric measurements } \\
\hline \multicolumn{8}{|l|}{ Weight $(\mathrm{kg}) \ddagger$} \\
\hline Mean & \multicolumn{2}{|c|}{$37 \cdot 1$} & \multicolumn{2}{|c|}{$36 \cdot 4$} & \multirow{2}{*}{\multicolumn{2}{|c|}{$\begin{array}{l}37.5 \\
10.0\end{array}$}} & \multirow{2}{*}{0.53} \\
\hline SD & \multicolumn{2}{|c|}{$10 \cdot 0$} & \multicolumn{2}{|c|}{$10 \cdot 1$} & & & \\
\hline \multicolumn{8}{|l|}{ BMI Z-score§ } \\
\hline Mean & \multirow{2}{*}{\multicolumn{2}{|c|}{$\begin{array}{l}0.03 \\
0.94\end{array}$}} & \multicolumn{2}{|c|}{0.25} & & & \multirow[t]{2}{*}{0.01} \\
\hline SD & & & & & & & \\
\hline \multicolumn{8}{|l|}{ WC $(\mathrm{cm}) \ddagger$} \\
\hline Mean & \multirow{2}{*}{\multicolumn{2}{|c|}{$\begin{array}{r}62 \cdot 8 \\
7.0\end{array}$}} & \multicolumn{2}{|c|}{$63 \cdot 1$} & & & \multirow{2}{*}{0.57} \\
\hline $\mathrm{SD}$ & & & & & & & \\
\hline \multicolumn{8}{|l|}{ School grade } \\
\hline Primary school, grades 3-4 (8-10 years) & 60 & 41.4 & 32 & $46 \cdot 4$ & 28 & $36 \cdot 8$ & \multirow[t]{4}{*}{0.06} \\
\hline Primary school, grades $5-6$ (10-12 years) & 53 & $36 \cdot 6$ & 24 & 34.8 & 29 & 38.2 & \\
\hline Middle school, grades $7-9$ ( $12-15$ years) & 29 & $20 \cdot 0$ & 10 & 14.5 & 19 & $25 \cdot 0$ & \\
\hline Otherll & 3 & $2 \cdot 1$ & 3 & $4 \cdot 3$ & 0 & 0 & \\
\hline \multicolumn{8}{|l|}{ Ethnicity } \\
\hline Caucasian & 127 & $87 \cdot 6$ & 58 & $84 \cdot 1$ & 69 & $90 \cdot 8$ & \multirow[t]{2}{*}{0.22} \\
\hline Otherq & 18 & 12.4 & 11 & $15 \cdot 9$ & 7 & $9 \cdot 2$ & \\
\hline
\end{tabular}

BMI, Body Mass Index; WC, waist circumference.

${ }^{*} P$ values indicate gender differences.

$\dagger n 143$.

$\neq n 142$.

$\S n 138$.

IIParticular path.

IFor boys, $n 0$ First Nation, $n 3$ Asians, $n 2$ Afro-Canadians, $n 1$ Latino-Canadian, $n 5$ other ethnicity. For girls, $n 1$ First Nation, $n 2$ Asians, $n 1$ Afro-Canadian, $n 0$ LatinoCanadian, $n 3$ other ethnicity.

Data are presented means (SD) or otherwise specified.

confirmatory factor analysis of the three-factor and twentyone-item model was calculated and set to 119 participants required for a statistical power level of $80 \%$, a significance level of 0.05 and an anticipated effect size of $0 \cdot 3^{(51)}$. This sample size was also similar to previous CTFEQ validation studies (Steff et al., $n$ 153 $3^{(24)}$; Yabsley et al., $n 158^{(26)}$ ). However, to consider missing data or absences from school at the second data collection, we planned to recruit about $160-165$ participants.

\section{Results}

\section{Descriptive analysis}

The mean age of participants was 11.0 (SD 1.9) years (Table 1). The majority of the participants were girls and Caucasians. The mean BMI $Z$-score was 0.03 (SD 0.94) and was significantly different for girls in comparison to boys $(P=0 \cdot 01)$.

\section{Factorial structure}

All three models tested in confirmatory factor analysis had an excellent fit to the data, meeting the recommended thresholds for each fit index as indicated by NonNormed Fit Index and Comparative Fit Index above 0.95, Root Mean Squared Error of Approximation below 0.06 and $\chi^{2} / \mathrm{df}$ below 2 (Table 2 ). Model 3 demonstrated a weaker fit to the data compared with the two other models and therefore was not retained. Model 1 had a lower AIC value $(-195 \cdot 38)$ than Model $2(-126 \cdot 05)$. No weak items for all three models were identified, with all standardised factor loadings being above $0 \cdot 3$ (see Table 3 for Model 2, results not shown for Models 1 and 3). However, Models 1 and 3 had one weaker factor loading under 0.4 (items 17 and 18, respectively), while all factor loadings of Model 2 were greater than $0 \cdot 4$.

\section{Reliability}

For Model 1, Cronbach's $\alpha$ coefficients were all adequate $(0.78,0.88$ and 0.90 for $\mathrm{CR}, \mathrm{UE}$ and $\mathrm{EE}$, respectively). In Model 2, the Cronbach's $\alpha$ coefficient for CR increased from 0.78 to 0.81 following the removal of items 17,18 and 21 (Table 3). The coefficients for UE, in which item 19 was removed, and EE both remained the same (Table 3). Test-retest reliability for Model 1 was poor to moderate for CR and EE (ICC $=0.48$; (95\% CI 0.35, 0.61) and ICC $=0.50 ;(95 \%$ CI 0.38, 0.62), respectively) and moderate to good for UE (ICC $=0.77 ; 95 \%$ CI 0.70, 0.84)). ICC for both CR and UE increased in Model 2 (ICC $=0.59$; $(95 \%$ CI $0.48,0.70)$ and ICC $=0.78$; (95\% CI 0.70, 0.84), respectively) and remained the same for EE (Table 3), therefore demonstrating a superior test-retest reliability for the seventeen-item model compared with the twenty-one-item 
Table 2 Fit indices for the three models of the CTFEQ evaluated by CFA

\begin{tabular}{|c|c|c|c|c|c|c|c|c|c|}
\hline Model & Items & Factors & $\chi^{2}(\mathrm{df})$ & $\chi^{2} / \mathrm{df}$ & NNFI & $\mathrm{CFI}$ & RMSEA & $90 \% \mathrm{Cl}$ & AIC \\
\hline Model 1 (Martín-Garcí & 21 & 3 & $176.621(186)(P=0.7)$ & 0.95 & 1.004 & 1.000 & 0.000 & $0.000,0.031$ & $-195 \cdot 379$ \\
\hline Model 2 (Bryant et al., 2018) & 17 & 3 & $105.950(116)(P=0.7)$ & 0.91 & 1.005 & 1.000 & 0.000 & $0.000,0.032$ & $-126 \cdot 050$ \\
\hline Model 3 (Yabsley et al., 2018) & 20 & 4 & $190.551(164)(P=0.1)$ & $1 \cdot 16$ & 0.957 & 0.963 & 0.034 & $0.000,0.053$ & -137.449 \\
\hline
\end{tabular}

CFA, confirmatory factor analysis; df, degrees of freedom; NNFI, Non-Normed Fit Index; CFI, Comparative Fit Index; RMSEA, Root Mean Squared Error of Approximation; AIC, Akaike's Information Criteria.

$n 142$.

Table 3 Factor loadings evaluated by CFA, internal consistency (Cronbach's $\alpha$ ), test-retest reliability (intra-class correlation coefficients) and descriptive statistics (mean \pm SD) for the retained CTFEQr17

\begin{tabular}{|c|c|c|c|c|c|c|c|c|c|c|c|c|}
\hline \multirow[b]{2}{*}{ Factors } & \multirow[b]{2}{*}{ Items } & \multirow[b]{2}{*}{ Factor loadings } & \multirow[b]{2}{*}{ Cronbach's $\alpha$} & \multirow[b]{2}{*}{ Test-retest ICC } & \multirow[b]{2}{*}{$95 \% \mathrm{Cl}$} & \multicolumn{2}{|c|}{$\begin{array}{c}\text { Total } \\
(n=145)\end{array}$} & \multicolumn{2}{|c|}{$\begin{array}{c}\text { Boys } \\
(n=69)\end{array}$} & \multicolumn{2}{|c|}{$\begin{array}{c}\text { Girls } \\
(n=76)\end{array}$} & \multirow[b]{2}{*}{$P^{*}$} \\
\hline & & & & & & Mean & SD & Mean & SD & Mean & SD & \\
\hline Cognitive restraint & $\begin{array}{r}1 \\
5 \\
11\end{array}$ & $\begin{array}{l}0.683 \\
0.646 \\
0.965\end{array}$ & 0.81 & 0.59 & $0.48,0.70$ & $1 \cdot 8$ & 0.7 & $1.9 \dagger$ & 0.7 & $1.7 \dagger$ & 0.7 & 0.04 \\
\hline Uncontrolled eating & $\begin{array}{r}3 \\
6 \\
8 \\
9 \\
12 \\
13 \\
15 \\
20\end{array}$ & $\begin{array}{l}0.816 \\
0.703 \\
0.766 \\
0.516 \\
0.750 \\
0.680 \\
0.785 \\
0.489\end{array}$ & 0.88 & 0.78 & $0.70,0.84$ & $2 \cdot 3$ & 0.7 & $2 \cdot 2$ & 0.7 & $2 \cdot 4$ & 0.7 & 0.1 \\
\hline Emotional eating & $\begin{array}{r}2 \\
4 \\
7 \\
10 \\
14 \\
16\end{array}$ & $\begin{array}{l}0.689 \\
0.714 \\
0.825 \\
0.796 \\
0.825 \\
0.808\end{array}$ & 0.90 & 0.50 & $0.38,0.62$ & 1.4 & 0.5 & $1 \cdot 3 \ddagger$ & 0.4 & $1.5 \ddagger$ & 0.6 & 0.03 \\
\hline
\end{tabular}

CFA, confirmatory factor analysis; ICC, intra-class correlation coefficient.

${ }^{\star} P$ values indicate gender differences.

†Boys had significantly higher CR than girls.

$\ddagger$ Girls had significantly higher EE than boys.

Student's $t$ test for differences between boys and girls.

$n 145$.

model (Model 1). Thus, the Model 2, comprising seventeen items and three factors as validated by Bryant and colleagues (CTFEQr17) ${ }^{(25)}$, was selected since it was offering an excellent model fit and a superior internal reliability and test-retest reliability compared to Model 1, especially for the CR factor.

\section{Construct validity}

Boys had slightly but significantly higher CR than girls $(P=0.04)$ and girls had slightly but significantly higher EE than boys ( $P=0.03)$ (Table 3$)$. CR correlated negatively with age for boys and girls $(r=-0 \cdot 37, P=0.002 ; r=-0.24$, $P=0.04$, respectively). UE correlated negatively with age for girls only $(r=-0 \cdot 34, P=0 \cdot 003)$. No correlation between EE and age, separated by sex, was found.

As presented in Table 4 , there was a positive correlation between UE and EE $(r=0.61, P<0.0001)$, but neither UE nor EE correlated with CR. CR was the only factor to correlate significantly with EAT-26 score $(r=0.43, P<0.0001)$. UE and EE correlated negatively with BMI $Z$-score $(r=-0 \cdot 26, P=0.003$ and $r=-0 ; \cdot 19, P=0 \cdot 03$, respectively). Only UE correlated negatively with WC $(r=-0 \cdot 21$, $P=0 \cdot 01)$ even though a tendency towards a negative correlation with WC can be observed with EE $(r=-0 \cdot 16$, $P=0.07)$. CR correlated positively with the percentage of EI from protein $(r=0.18, P=0.04)$ and with NutrientRich Food Index $9.3(r=0 \cdot 20, P=0.02)$ before adjustments were made for misreporting. After adjustments for misreporting, correlation with dietary intakes and diet quality did not change. For both methods, a trend towards a negative correlation between $\mathrm{CR}$ and EI was obtained $(r=-0 \cdot 16, P=0.07$ and $r=-0.17, P=0.06$, without and with adjustments for misreporting, respectively).

\section{Discussion}

The main objective of this study was to produce and validate the French version of the Child Three-Factor Eating Questionnaire ${ }^{(25)}$ in a French-speaking sample of 
Table 4 Correlations between the retained CTFEQr17 factors and eating attitudes, anthropometrics, dietary intake and diet quality*

\begin{tabular}{|c|c|c|c|c|c|c|}
\hline \multirow[b]{2}{*}{ Variable } & \multicolumn{2}{|c|}{ Cognitive restraint } & \multicolumn{2}{|c|}{ Uncontrolled eating } & \multicolumn{2}{|c|}{ Emotional eating } \\
\hline & $r$ & $P$ & $r$ & $P$ & $r$ & $P$ \\
\hline \multicolumn{7}{|l|}{ Eating behaviours } \\
\hline Cognitive restraint & 1 & & & & & \\
\hline Uncontrolled eating & $0 \cdot 10$ & 0.2 & 1 & & & \\
\hline Emotional eating & 0.14 & 0.1 & 0.61 & $<0.0001$ & 1 & \\
\hline EAT-26 & 0.43 & $<0.0001$ & 0.13 & 0.1 & 0.14 & 0.1 \\
\hline \multicolumn{7}{|c|}{ Anthropometric measurements } \\
\hline BMI Z-score & 0.07 & 0.4 & -0.26 & 0.003 & -0.19 & 0.03 \\
\hline $\mathrm{WC}, \mathrm{cm}$ & 0.05 & 0.6 & -0.21 & 0.01 & -0.16 & 0.07 \\
\hline \multicolumn{7}{|c|}{ Dietary intake and diet quality } \\
\hline Energy intake, kcal & -0.16 & 0.07 & -0.05 & 0.5 & -0.09 & 0.3 \\
\hline$\%$ carbohydrates & 0.01 & 0.95 & 0.00 & 0.99 & -0.01 & 0.9 \\
\hline$\%$ protein & 0.18 & 0.04 & -0.01 & 0.9 & -0.05 & 0.6 \\
\hline$\%$ lipids & -0.08 & 0.3 & -0.00 & 0.96 & 0.03 & 0.7 \\
\hline NRF 9.3 & 0.20 & 0.02 & -0.11 & 0.2 & -0.03 & 0.7 \\
\hline \multicolumn{7}{|c|}{ Dietary intake and diet quality† } \\
\hline Energy intake, kcalt & -0.17 & 0.06 & -0.07 & 0.5 & 0.01 & 0.9 \\
\hline$\%$ carbohydratest & -0.01 & 0.9 & 0.03 & 0.8 & -0.02 & 0.8 \\
\hline$\%$ proteint & 0.19 & 0.03 & -0.02 & $0 \cdot 8$ & -0.05 & 0.6 \\
\hline$\%$ lipids† & -0.08 & 0.4 & -0.03 & 0.7 & 0.04 & 0.7 \\
\hline NRF 9.3† & 0.18 & 0.04 & -0.12 & 0.2 & -0.05 & 0.5 \\
\hline
\end{tabular}

EAT-26, Eating Attitudes Test; BMI, Body Mass Index; WC, waist circumference; NRF 9.3, Nutrient-Rich Foods Index 9.3.

${ }^{*}$ Correlations determined with partial Pearson's correlation coefficients adjusted for sex and age.

†Values adjusted for misreporting.

Canadian children and adolescents aged 8-15 years. Results suggest that the French version of the seventeenitem model CTFEQ (CTFEQr17) confers an excellent factor structure and internal consistency, as well as moderate to adequate test-retest reliability in young French-speaking children and adolescents. As expected, CR correlated to EAT-26 score, but not with EI or WC and BMI $Z$-score thus confirming partly our hypothesis. It was also correlated with the proportion of EI from protein and diet quality. UE and EE correlated negatively only with BMI $Z$-score, the opposite of what was expected.

\section{Factorial structure and reliability}

The present study demonstrated that the model including seventeen items and three factors (CTFEQr17) had a superior model fit in a sample of French-speaking Canadian children and adolescents compared to other models found in the literature (twenty items, four factors and twenty-one items, three factors) ${ }^{(23,24,26)}$, therefore reproducing the results obtained by Bryant and colleagues ${ }^{(25)}$. CR factor showed weaker internal consistency in comparison to UE and EE. This has also been shown in the Spanish ${ }^{(23)}$, English ${ }^{(25)}$, Romanian ${ }^{(24)}$ and Turkish ${ }^{(27)}$ versions of the CTFEQ. In this study, both internal consistency and test-retest reliability of the questionnaire increased after the removal of items 17, 18 and 21 of the CR factor (Model 2), suggesting that some items in this factor may be less understood by the children. In other studies, items $17,18^{(25)}$ or $21^{(25,26)}$ of the CR factor have also been removed from the original factor structure due to weaker fit, thus improving validity ${ }^{(25,26)}$. However, the Spanish ${ }^{(23)}$ and Romanian $^{(24)}$ versions of the CTFEQ found a better fit with the complete questionnaire, retaining all six items comprising $\mathrm{CR}^{(23,24)}$. In adult populations, items 17,18 and 21 have also been shown to be problematic in one previous validation study among Canadian and American populations ${ }^{(17)}$, but most studies demonstrated a strong CR factor, retaining its six items ${ }^{(15,16,21)}$. One study among French adults and adolescents observed a weaker CR factor, but still preserved it ${ }^{(52)}$, while other studies among adolescents showed a weaker internal consistency of the CR factor compared to UE and EE factors ${ }^{53,54)}$. These results suggest that the six-item CR factor could be weaker in comparison to UE and EE, but this factor was judged to be adequate in the present study after the removal of items 17, 18 and 21, as in the model proposed by Bryant et $a l^{(25)}$.

\section{Construct validity}

The results of this study suggest that eating behaviour traits among children and adolescents may be influenced by sex and age. Boys had a higher CR than girls. However, previous studies have shown the opposite result ${ }^{(52,55)}$. This study also showed that girls reported a higher EE than boys, which is supported by other studies ${ }^{(52,55,56)}$. This may indicate that girls respond more to emotions to influence their eating habits compared to boys. Other studies did not demonstrate any differences between sexes at this age ${ }^{(23,26)}$, while some observed a significant difference for $\mathrm{UE}^{(25,52)}$, which highlights possible differences in samples used related to sex. According to Cohen's effect size ${ }^{(57)}$, the strength of these correlations represented a small effect size for CR $(r=0 \cdot 29)$ and medium effect size for EE $(r=0 \cdot 39)$, indicating that these correlations might not be clinically 
meaningful. Age may also be involved in influencing eating behaviours. Accordingly, in this study, CR was negatively correlated with age in boys and girls and UE was negatively correlated with age in girls only. These correlations are supported by other studies among children and adolescents, for $\mathrm{CR}^{(25,26)}$ and $\mathrm{UE}^{(23,25,26)}$. Younger individuals may be sensitive to parental eating practices and children's eating behaviours are influenced by the parent's own eating behaviours ${ }^{(58)}$. Therefore, younger individuals might be more sensitive to their parent's pressures to control their food intake which may influence their eating behaviours, like CR, while adolescents might be less likely to be under the direct influence of their parents regarding their eating behaviours. Our results also show that younger individuals may be more sensitive to internal or environmental stimuli about how they eat. As in this study, no correlation between age and EE was observed in previous studies ${ }^{(23,25,26)}$.

The present study shows a moderate positive correlation between UE and EE. The CR factor did not significantly correlate with any other factor. Both of these results are in line with the English and Romanian versions of the questionnaire ${ }^{(24,25)}$ but contradictory to the Spanish version, which demonstrated a negative correlation between UE and $\mathrm{CR}^{(23)}$ and the English version in a Canadian sample, which showed a positive correlation between $\mathrm{EE}$ and $\mathrm{CR}^{(26)}$. However, both of these versions also observed a positive correlation between $\mathrm{UE}$ and $\mathrm{EE}^{(23,26)}$. As $\mathrm{UE}$ and $\mathrm{EE}$ derive from disinhibition and susceptibility to hunger in the original TFEQ and imply overeating, this correlation is therefore expected. While restraint has been known to be correlated with overeating ${ }^{(59)}$, it has also been previously shown that disinhibition can be present independently of restraint ${ }^{(60)}$, therefore supporting the lack of correlation between CR and UE or EE in this study. The disparity in results for CR correlations with other eating behaviours may be the result of cultural or social differences in the samples studied. Further studies are needed to understand the relationship between CR and overeating in children populations.

This study observed a moderate positive correlation between CR and EAT-26, which characterises the presence of symptoms associated with eating disorders. Compared to non-restrained eaters, restrained eaters are more likely to express disordered eating patterns ${ }^{(61)}$. Previous studies have also shown positive correlations between disordered eating attitudes (EAT-26) and restrained eating ${ }^{(62-65)}$, which supports these results.

The negative correlations between both UE and EE factors and BMI $Z$-scores and between UE and WC are supported by a previous study demonstrating an inverse correlation between UE and EE and BMI $Z$-scores, but only in boys ${ }^{(26)}$, and other studies demonstrating a negative correlation between $\mathrm{UE}$ and $\mathrm{BMI}^{(23)}$ and UE being significantly lower among children and adolescents with obesity or overweight compared to those of normal weight ${ }^{(27)}$. In contrast to those results, positive correlations have been shown between both UE and $\mathrm{EE}$ and body weight and $\mathrm{BMI}^{(24)}$.
Among adolescents, EE and UE have also been shown to be positively correlated with BMI, assessed with the adult TFEQ-R18 ${ }^{(21,53)}$, while disinhibition has been positively correlated with BMI in children ${ }^{(22,66)}$ and adolescents ${ }^{(67-69)}$. Overall, these results may imply that the young normal weight individuals reporting higher UE and EE are at higher risk for future weight gain. Longitudinal studies are needed in children to investigate these correlations. To help better understand these results, future studies assessing the CTFEQr17 in relation to eating behaviours and anthropometric measures should consider a larger sample size and life stage (e.g. childhood, adolescence, adulthood).

Currently, no studies among children and adolescents have evaluated the correlations between dietary intake, diet quality and eating behaviour traits as measured with the CTFEQ. In this study, CR was positively correlated with the proportion of energy derived from protein and diet quality, both with and without adjustments for misreporting. Among female teenagers and young adults ${ }^{(52)}$ and children and adults ${ }^{(56)}$, a higher proportion of energy derived from protein was also correlated with $\mathrm{CR}$, but both studies also observed a negative correlation between $\mathrm{EI}$ and $\mathrm{CR}^{(52,56)}$, which was not shown in the present study although a tendency towards this correlation was obtained. Another study among young girls observed that at ages 7 and 9 years, restraint was negatively correlated with $\mathrm{EI}^{(70)}$. It could be possible that the single 24-h dietary recall used in this study may not be enough to observe under-consumption since correlations between CR and lower EI were NS. With regard to diet quality, a negative correlation between CR and the consumption of energy-dense foods was observed among teenagers and young adults ${ }^{(52)}$ and a positive correlation between restraint and both the consumption of fruits and vegetables and eating fast food less frequently was observed among young adult men ${ }^{(71)}$, which supports our results. This suggests that restrained individuals tend to choose foods of higher nutritional quality and richer in protein, perhaps to influence their body weight and their satiety. Regarding UE and EE, they have been previously positively correlated with $\mathrm{EI}^{(52)}$. Other studies observed that children with high UE had a greater preference for high-fat foods ${ }^{(25,26)}$. The present study did not observe any correlation between dietary intakes and UE or EE, perhaps due to its single measured observation of food intake.

\section{Strengths and limitations}

A strength of this study was the evaluation of test-retest reliability, a measurement that allows the adequate assessment of temporal stability of the results obtained by the same person. Also, an assessment of children's dietary intakes rather than food preferences was included, in contrast to previous studies $^{(25,26)}$. The 24 -h dietary recall allowed the acquirement of more precise information on the actual intakes of the participants. One limitation of this study is that the pubertal stage via Tanner staging was not evaluated, which has 
been shown to influence eating behaviour traits in previous work $^{(23)}$. Furthermore, information on the socioeconomic level of participants was not collected which may also influence study outcomes since correlations between eating behaviour traits and socioeconomic level have previously been shown among adolescents ${ }^{(55)}$ and adults ${ }^{(72-74)}$. Additionally, although the 24-h dietary recall is a well-established method to assess children's dietary intake ${ }^{(75)}$, the present study only collected one 24-h dietary recall during the first visit. Usually, repeated recalls should be collected to assess the population distribution of usual intakes ${ }^{(76)}$ and many studies use repeated 24-h dietary recall instead of a single observation ${ }^{(77)}$. As with any dietary assessment method, individuals with overweight and obesity ${ }^{(75-81)}$, who engage in higher screen time or higher moderate to vigorous physical activity ${ }^{(79)}$, who have higher sedentary behavior or lower socio-economic status ${ }^{(81)}$ and adolescent girls $^{(77)}$ may misreport their food intake. Under-reporting of dietary intakes has also been shown to increase with age among children ${ }^{(77)}$ and to be higher among restrained adolescents ${ }^{(79)}$, which justifies adjustments for misreporting. However, it is important to acknowledge the fact that the adjustment method assumes weight stability ${ }^{(46)}$ and does not distinguish individuals who truly under-eat or over-eat from those who are under-reporting or over-reporting their intakes which may bias the results ${ }^{(79,81)}$.

\section{Conclusion}

The CTFEQr17 is a valid and reliable tool for assessing eating behaviour traits among French-Speaking Canadian children and adolescents. This questionnaire could represent an important assessment tool to better understand childhood obesity and help in the development of strategies and interventions aimed to prevent unhealthy eating behaviour traits and childhood obesity.

\section{Acknowledgements}

Acknowledgements: We thank the schools for their availability and their interest to participate in this research. We also acknowledge the help of Francine Pérusse for her role in the coordination of data collection. We also thank Diane Drolet, Manon-Bordeleau, Laurence Deslauriers, Maurine Dubeuf, Corentin Clemot, Sabrina Labrecque and Lucie Allard for data collection. We also thank Eric Frenette, Joël Gagnon-and Christian Couture for their help with statistical analysis. I.F. is a recipient of a M.Sc. scholarship from the Canadian Institutes of Health Research. R.J. is a recipient of a Ph.D. scholarship from the Canadian Institutes of Health Research (Fellowship number: 430872). Financial support: This study was funded by the Québec Heart and Lung
Institute. Conflict of interest: There are no conflicts of interest. Authorship: V.D. and E.J.B. designed the research. V.D., I.F., R.J., S.P. and D.L. participated in the data collection. I.F., R.J., V.D. and S.P. analysed and interpreted the data. I.F. wrote the first draft of the manuscript. All of the authors revised the manuscript and approved the final version. Ethics of human subject participation: This study was conducted according to the guidelines from the Declaration of Helsinki and all procedures involving research study participants were approved by the Research Ethics Committee of the Québec Heart and Lung Institute. Written informed consent was obtained from all participants and parents or guardians.

\section{Supplementary material}

For supplementary material accompanying this paper visit https://doi.org/10.1017/S136898002100392X

\section{References}

1. Statistics Canada (2017) Measured Children and Youth Body Mass Index (BMI) (World Health Organization Classification), by Age Group and Sex, Canada and Provinces, Canadian Community Health Survey - Nutrition. https://www150. statcan.gc.ca/t1/tbl1/en/tv.action?pid=1310079501 (accessed March 2020).

2. Kral TV (2016) Eating behaviors and weight development in obesity-prone children and the importance of the research of Albert J. Stunkard. Curr Obes Rep 5, 126-131.

3. Kelsey MM, Zaepfel A, Bjornstad P et al. (2014) Age-related consequences of childhood obesity. Gerontology $\mathbf{6 0}$, 222-228.

4. Serdula MK, Ivery D, Coates RJ et al. (1993) Do obese children become obese adults? A review of the literature. Prev Med 22, 167-177.

5. Kumar S \& Kelly AS (2017) Review of childhood obesity: from epidemiology, etiology, and comorbidities to clinical assessment and treatment. Mayo Clin Proc 92, 251-265.

6. Roblin L (2007) Childhood obesity: food, nutrient, and eating-habit trends and influences. Appl Physiol Nutr Metab 32, 635-645.

7. Gurnani M, Birken C \& Hamilton J (2015) Childhood obesity: causes, consequences, and management. Pediatr Clin North Am 62, 821-840.

8. Bryant EJ, Rehman J, Pepper LB et al. (2019) Obesity and eating disturbance: the role of TFEQ restraint and disinhibition. Curr Obes Rep 8, 363-372.

9. Jacob R, Drapeau V, Tremblay A et al. (2018) The role of eating behavior traits in mediating genetic susceptibility to obesity. Am J Clin Nutr 108, 445-452.

10. Chaput JP, Leblanc C, Perusse L et al. (2009) Risk factors for adult overweight and obesity in the Quebec family study: have we been barking up the wrong tree? Obesity 17, 1964-1970.

11. Drapeau V, Provencher V, Lemieux S et al. (2003) Do 6-year changes in eating behaviors predict changes in body weight? Results from the Quebec family study. Int J Obes Relat Metab Disord 27, 808-814.

12. Power TG, Hidalgo-Mendez J, Fisher JO et al. (2020) Obesity risk in Hispanic children: bidirectional associations between child eating behavior and child weight status over time. Eat Behav 36, 101366. 
13. Miguet M, Masurier J, Chaput JP et al. (2019) Cognitive restriction accentuates the increased energy intake response to a 10 -month multidisciplinary weight loss program in adolescents with obesity. Appetite 134, 125-134.

14. Stunkard AJ \& Messick S (1985) The three-factor eating questionnaire to measure dietary restraint, disinhibition and hunger. J Psychosom Res 29, 71-83.

15. Karlsson J, Persson LO, Sjostrom L et al. (2000) Psychometric properties and factor structure of the three-factor eating questionnaire (TFEQ) in obese men and women. Results from the Swedish obese subjects (SOS) study. Int J Obes Relat Metab Disord 24, 1715-1725.

16. Tholin S, Rasmussen F, Tynelius P et al. (2005) Genetic and environmental influences on eating behavior: the Swedish young male twins study. Am J Clin Nutr 81, 564-569.

17. Cappelleri JC, Bushmakin AG, Gerber RA et al. (2009) Psychometric analysis of the three-factor eating questionnaire-R21: results from a large diverse sample of obese and non-obese participants. Int J Obes 33, 611-620.

18. Loffler A, Luck T, Then FS et al. (2015) Eating behaviour in the general population: an analysis of the factor structure of the German version of the three-factor-eating-questionnaire (TFEQ) and its association with the body mass index. PLoS One 10, e0133977.

19. Keskitalo K, Tuorila H, Spector TD et al. (2008) The threefactor eating questionnaire, body mass index, and responses to sweet and salty fatty foods: a twin study of genetic and environmental associations. Am J Clin Nutr 88, 263-271.

20. de Medeiros ACQ, Yamamoto ME, Pedrosa LFC et al. (2017) The Brazilian version of the three-factor eating questionnaire-R21: psychometric evaluation and scoring pattern. Eat Weight Disord 22, 169-175.

21. Angle S, Engblom J, Eriksson T et al. (2009) Three factor eating questionnaire-R18 as a measure of cognitive restraint, uncontrolled eating and emotional eating in a sample of young Finnish females. Int J Behav Nutr Phys Act 6, 41.

22. Rutters F, Nieuwenhuizen AG, Vogels N et al. (2008) Leptinadiposity relationship changes, plus behavioral and parental factors, are involved in the development of body weight in a Dutch children cohort. Physiol Behav 93, 967-974.

23. Martin-Garcia M, Vila-Maldonado S, Rodriguez-Gomez I et al. (2016) The Spanish version of the three factor eating questionnaire-R21 for children and adolescents (TFEQ-R21C): psychometric analysis and relationships with body composition and fitness variables. Physiol Behav 165, 350-357.

24. Steff M, Verney J, Marinau M et al. (2019) Toward a Romanian version of the three-factor eating questionnaireR21 for children and adolescents (CTFEQ-R21): preliminary psychometric analysis and relation with body composition. Dev Period Med 23, 45-53.

25. Bryant EJ, Thivel D, Chaput JP et al. (2018) Development and validation of the child three-factor eating questionnaire (CTFEQr17). Public Health Nutr 21, 2558-2567.

26. Yabsley JL, Gunnell KE, Bryant EJ et al. (2018) Validation of a child version of the three-factor eating questionnaire in a Canadian sample: a psychometric tool for the evaluation of eating behaviour. Public Health Nutr 22, 431-443.

27. Demir D, Bektas M, Bektas I et al. (2020) Psychometric properties of the Turkish version of the child three-factor eating questionnaire for primary and secondary school students. Public Health Nutr 24, 427-435.

28. Leichner P, Steiger H, Puentes-Neuman G et al. (1994) Validation of an eating attitude scale in a French-speaking Quebec population. Can J Psychiatry 39, 49-54.

29. Garner DM, Olmsted MP, Bohr Y et al. (1982) The eating attitudes test: psychometric features and clinical correlates. Psychol Med 12, 871-878.

30. Must A \& Anderson SE (2006) Body mass index in children and adolescents: considerations for population-based applications. Int J Obes 30, 590-594.
31. de Onis M, Onyango AW, Borghi E et al. (2007) Development of a WHO growth reference for school-aged children and adolescents. Bull World Health Organ 85, 660-667.

32. Sharma A \& Metzger DL (2020) Calculator: WHO Anthropometric Z-Scores 0-9 Years. https://apps.cpeggcep.net/quickZ_WHO/ (accessed August 2019).

33. Steinfeldt L, Anand J \& Murayi T (2013) Food reporting patterns in the USDA automated multiple-pass method. Procedia Food Sci 2, 145-156.

34. Raper N, Perloff B, Ingwersen L et al. (2004) An overview of USDA's dietary intake data system. J Food Comp Anal 17, 545-555.

35. Health Products and Foods Branch (2015) Nutrition Research Division. Canadian Nutrient File (CNF). https://foodnutrition.canada.ca/cnf-fce/index-eng.jsp (accessed March 2019).

36. Fulgoni VL 3rd, Keast DR \& Drewnowski A (2009) Development and validation of the nutrient-rich foods index: a tool to measure nutritional quality of foods. $J$ Nutr 139, 1549-1554.

37. Drewnowski A \& Fulgoni V 3rd (2008) Nutrient profiling of foods: creating a nutrient-rich food index. Nutr Rev 66, 2339.

38. Cheema JR (2014) A review of missing data handling methods in education research. Rev Educ Res 84, 487-508.

39. Hu L \& Bentler P (1999) Cutoff criteria for fit indexes in covariance structure analysis: conventional criteria versus new alternatives. Struct Equ Modeling 6, 1-55.

40. Byrne B (2010) Structural Equation Modeling with AMOS: Basic Concepts, Applications, and Programming, 2nd ed. New York: Routledge Taylor \& Francis Group.

41. Bollen KA (1989) Structural Equations with Latent Variables. New York: Wiley.

42. Hair JF (2010) Multivariate Data Analysis, 7th ed. Upper Saddle River, NJ; London: Prentice Hall.

43. Kline RB (2011) Principles and Practice of Structural Equation Modeling, 3rd ed. New York: Guilford Press.

44. Spiegelman D \& Hertzmark E (2010) The SAS ICC9 Macro. https://cdn1.sph.harvard.edu/wp-content/uploads/sites/271/ 2012/09/icc9.pdf (accessed August 2019).

45. Koo TK \& Li MY (2016) A guideline of selecting and reporting intraclass correlation coefficients for reliability research. $J$ Chiropr Med 15, 155-163.

46. Huang TT, Roberts SB, Howarth NC et al. (2005) Effect of screening out implausible energy intake reports on relationships between diet and BMI. Obes Res 13, 1205-1217.

47. Institute of Medicine (2002) Dietary Reference Intakes for Energy, Carbohydrate, Fiber, Fat, Fatty Acids, Cholesterol, Protein, and Amino Acids, Part I. Washington, DC: The National Academies Press.

48. Statistics Canada (2019) Physical Activity and Screen Time among Canadian Children and Youth, 2016 and 2017. Health Facts Sheets. https://www150.statcan.gc.ca/n1/ pub/82-625-x/2019001/article/00003-eng.htm （accessed March 2020).

49. Black AE (2000) Critical evaluation of energy intake using the Goldberg cut-off for energy intake: basal metabolic rate. A practical guide to its calculation, use and limitations. Int $J$ Obes Relat Metab Disord 24, 1119-1130.

50. Terwee CB, Bot SD, de Boer MR et al. (2007) Quality criteria were proposed for measurement properties of health status questionnaires. J Clin Epidemiol 60, 34-42.

51. Soper DS (2021) A Priori Sample Size Calculator for Structural Equation Models. http://www.danielsoper.com/statcalc (accessed May 2021).

52. de Lauzon B, Romon M, Deschamps V et al. (2004) The three-factor eating questionnaire-R18 is able to distinguish among different eating patterns in a general population. J Nutr 134, 2372-2380. 
53. Banna JC, Panizza CE, Boushey CJ et al. (2018) Association between cognitive restraint, uncontrolled eating, emotional eating and BMI and the amount of food wasted in early adolescent girls. Nutrients 10, 1279.

54. Megalakaki O, Mouveaux M, Hubin-Gayte M et al. (2013) Body image and cognitive restraint are risk factors for obesity in French adolescents. Eat Weight Disord 18, 289-295.

55. Snoek HM, van Strien T, Janssens JM et al. (2008) Restrained eating and BMI: a longitudinal study among adolescents. Health Psychol 27, 753-759.

56. Lluch A, Herbeth B, Mejean L et al. (2000) Dietary intakes, eating style and overweight in the Stanislas family study. Int J Obes Relat Metab Disord 24, 1493-1499.

57. Cohen J (1992) A power primer. Psychol Bull 112, 155-159.

58. Birch LL \& Davison KK (2001) Family environmental factors influencing the developing behavioral controls of food intake and childhood overweight. Pediatr Clin North Am 48, 893-907.

59. Polivy J \& Herman CP (2017) Restrained eating and food cues: recent findings and conclusions. Curr Obes Rep $\mathbf{6}$, 79-85.

60. Bryant EJ, King NA \& Blundell JE (2008) Disinhibition: its effects on appetite and weight regulation. Obes Rev 9, 409-419.

61. Johnson F, Pratt M \& Wardle J (2012) Dietary restraint and self-regulation in eating behavior. Int J Obes 36, 665-674.

62. Costarelli V \& Patsai A (2012) Academic examination stress increases disordered eating symptomatology in female university students. Eat Weight Disord 17, e164-e169.

63. Bryant EJ, Kiezebrink K, King NA et al. (2010) Interaction between disinhibition and restraint: implications for body weight and eating disturbance. Eat Weight Disord 15, e43-e51.

64. Cebolla A, Barrada JR, van Strien T et al. (2014) Validation of the Dutch eating behavior questionnaire (DEBQ) in a sample of Spanish women. Appetite 73, 58-64.

65. Siervo M, Boschi V, Papa A et al. (2005) Application of the SCOFF, eating attitude test 26 (EAT 26) and eating inventory (TFEQ) questionnaires in young women seeking diettherapy. Eat Weight Disord 10, 76-82.

66. Vogels N, Posthumus DL, Mariman EC et al. (2006) Determinants of overweight in a cohort of Dutch children. Am J Clin Nutr 84, 717-724.

67. Maayan L, Hoogendoorn C, Sweat V et al. (2011) Disinhibited eating in obese adolescents is associated with orbitofrontal volume reductions and executive dysfunction. Obesity 19, 1382-1387.
68. Gallant AR, Tremblay A, Perusse L et al. (2010) The three-factor eating questionnaire and BMI in adolescents: results from the Quebec family study. BrJ Nutr 104, 1074-1079.

69. Elfhag K \& Linne Y (2005) Gender differences in associations of eating pathology between mothers and their adolescent offspring. Obes Res 13, 1070-1076.

70. Shunk JA \& Birch LL (2004) Validity of dietary restraint among 5- to 9-year old girls. Appetite 42, 241-247.

71. Langlois F, Langlois MF, Carpentier AC et al. (2011) Ghrelin levels are associated with hunger as measured by the threefactor eating questionnaire in healthy young adults. Physiol Behav 104, 373-377.

72. Pigeyre M, Rousseaux J, Trouiller P et al. (2016) How obesity relates to socio-economic status: identification of eating behavior mediators. Int J Obes 40, 1794-801.

73. Pigeyre M, Duhamel A, Poulain JP et al. (2012) Influence of social factors on weight-related behaviors according to gender in the French adult population. Appetite 58, 703-709.

74. Dykes J, Brunner EJ, Martikainen PT et al. (2004) Socioeconomic gradient in body size and obesity among women: the role of dietary restraint, disinhibition and hunger in the Whitehall II study. Int J Obes Relat Metab Disord 28, 262-268.

75. Walker JL, Ardouin S \& Burrows T (2018) The validity of dietary assessment methods to accurately measure energy intake in children and adolescents who are overweight or obese: a systematic review. Eur J Clin Nutr 72, 185-97.

76. Moreno LA, Kersting M, de Henauw S et al. (2005) How to measure dietary intake and food habits in adolescence: the European perspective. Int J Obes 2, S66-S77.

77. Foster E \& Bradley J (2018) Methodological considerations and future insights for 24-h dietary recall assessment in children. Nutr Res 51, 1-11.

78. Murakami K \& Livingstone MB (2016) Prevalence and characteristics of misreporting of energy intake in US children and adolescents: National Health and Nutrition Examination Survey (NHANES) 2003-2012. Br J Nutr 115, 294-304.

79. Bel-Serrat S, Julian-Almarcegui C, Gonzalez-Gross $\mathrm{M}$ et al. (2016) Correlates of dietary energy misreporting among European adolescents: the healthy lifestyle in Europe by nutrition in adolescence (HELENA) study. Br J Nutr 115 , 1439-1452.

80. Livingstone MB \& Black AE (2003) Markers of the validity of reported energy intake. J Nutr 133, Suppl. 3, 895S-920S.

81. Lioret S, Touvier M, Balin M et al. (2011) Characteristics of energy under-reporting in children and adolescents. $\mathrm{Br} J$ Nutr 105, 1671-1680. 